\title{
Fundamental Architecture and Performance Analysis of Photofission Pulsed Space Propulsion System Using Ultra-Intense Laser
}

\author{
Robert LeMoyne', Timothy Mastroianni² \\ ${ }^{1}$ Independent (Senior Member AIAA \& Senior Member IEEE), Running Springs, California, USA \\ ${ }^{2}$ Independent, Pittsburgh, Pennsylvania, USA \\ Email: rlemoyne07@gmail.com
}

Received December 2014

\begin{abstract}
Photofission enables a unique capability for the domain of non-chemical space propulsion. An ultra-intense laser enables the capacity to induce nuclear fission through the development of bremsstrahlung photons. A fundamental architecture and performance analysis of a photofission pulsed space propulsion system through the operation of an ultra-intense laser is presented. A historical perspective of previous conceptual nuclear fission propulsion systems is addressed. These applications use neutron derived nuclear fission; however, there is inherent complexity that has precluded further development. The background of photofission is detailed. The conceptual architecture of photofission pulsed space propulsion and fundamental performance parameters are established. The implications are the energy source and ultra-intense laser can be situated far remote from the propulsion system. Advances in supporting laser technologies are anticipated to increase the potential for photofission pulsed space propulsion. The fundamental performance analysis of the photofission pulsed space propulsion system indicates the architecture is feasible for further evaluation.
\end{abstract}

\section{Keywords}

Ultra-Intense Laser, Photofission, Photofission Propulsion, Nuclear Fission, Space Propulsion, Pulsed Space Propulsion

\section{Introduction}

Non-chemical alternatives for space propulsion offer substantially reduced propellant requirements by contrast to standard chemical propulsion. Non-chemical propulsion systems span the domain of nuclear reactions. For example, LeMoyne and Mastroianni have successfully demonstrated multiple configurations for the realm of positron antimatter propulsion through the implementation of ultra-intense lasers, such as applications for pulsed space propulsion [1]-[4]. The objective of the research is to present a nuclear pulsed space propulsion system that utilizes photofission as a mechanism for imparting thermal energy upon the propulsive fluid. 
Nuclear fission offers another non-chemical propulsion system contingency. In general, nuclear fission involves the splitting of a nucleus into two separate nuclei of lesser atomic mass. The reaction is initiated by neutrons of sufficient energy to interact with the cross-section of a respective nucleus. For example, Equation (1) illustrates the nuclear fission reaction involving uranium-235 [5] [6].

$$
{ }_{0}^{1} n+{ }_{92}^{235} U \rightarrow{ }_{56}^{141} \mathrm{Ba}+{ }_{36}^{92} \mathrm{Kr}+3{ }_{0}^{1} n
$$

There are many permutations of the byproducts of a nuclear fission reaction. Equation (1) demonstrates a typical nuclear fission reaction. The reaction involves a series of events. First a neutron of appropriate kinetic energy interacts with a uranium-235 nucleus. Then the nucleus splits into two lighter byproduct nuclei, such as krypton-92 and barium-141, with the additional yield of three highly thermal neutrons. The nuclear fission reactions release a considerable amount of energy, which can be approximated by the associated mass defect of the reaction [5] [6].

\section{Historical Perspective of Nuclear Fission Derived Propulsion Systems}

The concept for developing propulsion systems that apply nuclear reactions, such as fission, has been previously considered. During the 1960's Los Alamos National Laboratory of the United States of America advanced the research, development, test, and evaluation for the Nuclear Engine for Rocket Vehicle Application (NERVA). The NERVA propulsion system features a fission nuclear reactor that provides an energy source to its propulsive fluid. Another associated Los Alamos National Laboratory endeavor that developed a prototype nuclear fission derived propulsion system named Kiwi was derived from Project Rover [7] [8].

Another concept involving uncontrolled nuclear energy was proposed with Project Orion. The Project Orion concept involves the detonation of a series of pulsed nuclear explosions to propel a spacecraft. The nuclear detonations would involve the use of nuclear fission and/or nuclear fusion [9].

There are many design issues implicit with these two strategies that incorporate uncontrolled nuclear reactions, such as Project Orion, and controlled nuclear energy, such as the NERVA and Project Rover. With respect to the Project Orion concept the danger of implementation is quite apparent. The intrinsic space ignited nuclear detonations must first be transported from a terrestrial setting to an orbital environment. Launch vehicle systems entail the inherent risk of exploding during launch. Encapsulating a payload to a launch vehicle consisting of an assembled or partially assembled nuclear bomb proposed in Project Orion would imply significant risk with regards to accidental nuclear detonation and the associated electromagnetic pulse throughout a wide span of the terrestrial flight path footprint.

Regarding the relatively safer controlled nuclear fission propulsion system, such as the NERVA, there is still an issue with controlling the nuclear fission reaction in a mass efficient manner. Typical nuclear reactors require cadmium, carbon, and/or heavy water (deuterium prevalent water) to moderate the neutron flux [10]. Heavy water, which is utilized by the Canadian-invented CANDU reactor, permits the moderation of neutrons and thermal regulation with tandem use of standard light water [11]. The implication of these multiple materials needed to moderate neutron flux for a conventional nuclear fission architecture inherently negatively impacts a propulsion system in terms of mass and complexity.

\section{Photofission Derived Propulsion, a New Perspective}

An alternative to utilizing neutron derived nuclear fission is the use of photofission as a mechanism to induce nuclear fission of a heavy nucleus. Photofission incorporates the use of ultra-intense lasers on the order of approximately $10^{20} \mathrm{~W} / \mathrm{cm}^{2}$ [12]-[14]. The phenomenon involves the generation of high energy electrons, bremsstrahlung photons, and nuclear interactions [13]. Previous architectures for positron-antimatter propulsion, which is a subset of non-chemical nuclear propulsion, advocating the role of ultra-intense lasers of roughly $10^{20} \mathrm{~W} / \mathrm{cm}^{2}$ have been presented by LeMoyne and Mastroianni [1]-[4]. Photofission applications have even been reduced to tabletop laser configurations [14].

The advantage of photofission is the inherent means of activating the fission event. Photofission is activated by an ultra-intense laser system rather than a neutron source. This paradigm shift involves commencement and sustained process of fission events to occur based on the controllable activity of the laser operation, instead of a difficult to regulate neutron flux. The inherent materials associated with neutron moderation could be consolidated. The energy source and ultra-intense laser can be remotely situated from the propulsion system. 
A photofission derived propulsion system would have the inherent advantages of high energy density and controllable energy yield. As an advantage to traditional nuclear fission strategies, the photofission derived propulsion system would also have reduced system complexity and mass. The envisioned photofission propulsion system would incorporate a pulsed space propulsion configuration with hydrogen as the propulsive fluid.

\section{Background of Photofission}

Photofission primarily differs from traditional nuclear fission with regards to the activating mechanism of the nuclear fission event. Rather than a neutron source, high energy gamma radiation excites the nucleus for a pending fission event, therefore the term photofission. For gamma radiation that is bound within the giant dipole resonance of the nucleus, deformation of the nucleus due to oscillatory motion occurs. Such deformation of the nucleus enables Coulomb repulsions to transcend strong nuclear force inducing fission [15].

Photofission is commenced by interaction between the nucleus and bremsstrahlung photons. The bremsstrahlung photons for inducing photofission have been attributed to a minimum threshold, such as approximately 5 $6 \mathrm{MeV}$ [15] [16]. Ultra-intense lasers on the order of approximately $10^{20} \mathrm{~W} / \mathrm{cm}^{2}$ have produced bremsstrahlung photons through the generation of laser initiated plasma. During 2000, two institutions with ultra-intense lasers systems achieved the objective of laser induced photofission [12]-[14]. The accomplishment was achieved using the NOVA laser of Lawrence Livermore National Laboratory with a yield of $7 \times 10^{4}$ fissions of uranium-238 per joule of laser energy [13] [14]. The VULCAN laser of Rutherford Appleton Laboratory produced a yield of $2 \times$ $10^{4}$ fissions of uranium-238 per joule of laser energy [12] [14]. Another configuration incorporating a tabletop laser capable of attaining ultra-intense laser thresholds yielded $10^{4}$ fissions per joule of laser energy with uranium-238 also as a target [14].

Schwoerer et al. applied a tabletop laser for the photofission of actinides. The experiments were conducted using a Jena 15 TW laser, which incorporates titanium-sapphire, chirped pulse amplification, and a maximum energy threshold of $1.2 \mathrm{~J}$ within the duration of $80 \mathrm{fs}$, and a repetition rate of $10 \mathrm{~Hz}$. A laser intensity of $10^{20}$ $\mathrm{W} / \mathrm{cm}^{2}$ is feasible in consideration of the laser beam's temporal quality that enables pulses to be targeted to an area less than $5 \mu \mathrm{m}^{2}[14]$.

The laser beam is targeted on to an intermediary material of two sheets of tantalum before the uranium-238 target. The first tantalum sheet is $50 \mu \mathrm{m}$ thick. At the first sheet a hot plasma is generated. The second tantalum sheet is $1 \mathrm{~mm}$ thick. The second thicker sheet functions as a bremsstrahlung converter, as electrons accelerated from the plasma of the first sheet collide with the second sheet [14].

A notable advantage of photofission is that demonstration experiments have been conducted using target isotopes that are naturally occurring and relatively abundant. For example, photofission has been conducted using thorium, the natural isotopes of uranium, and even lead [17] [18]. The implications of this diversity of potential candidates for photofission are that time consuming and resource intensive endeavors, such as isotope separation, are not necessary for the viable photofission of heavy nuclei.

The characterization of the mass yield distribution for photofission of uranium-238 is still an active and evolveing subject [19]. In order to derive performance parameters for a pulsed space propulsion system, the energy yield for photofission of uranium-238 is approximated to the equivalence of neutron induced fission of uranium-235, which has been thoroughly characterized over course of a half century of experimental physics. The approximate energy yield per fission event of uranium-235 is $200 \mathrm{MeV}$ [5].

\section{Conceptual Architecture of Photofission Pulsed Space Propulsion}

Pulsed space propulsion has been advocated as a practical implementation of various classes of non-chemical propulsion. For example, radioisotope decay pulsed space propulsion and positron antimatter pulsed space propulsion have been demonstrated from a conceptual architecture and fundamental performance analysis perspective. Pulsed space propulsion systems are relevant for the scope of the primary maneuvering and attitude control of small spacecraft. Propulsive fluid is progressively heated until a prescribed thermal threshold is attained for active propulsion. Contrary to steady state propulsion, the radioactive loads imparted by pulsed space propulsion are considerably reduced, mitigating adverse influence of ambient radiation [2] [20] [21].

The photofission space propulsion system incorporates a pulsed propulsion configuration. Thermal energy accumulated by photofission events heats the propulsive fluid to a prescribed thermal threshold. Upon achieving the thermal threshold, the photofission pulsed space propulsion system becomes active for generating thrust. 
A critical design issue is the selection of the propulsive fluid. Vulpetti advocated a liquid propellant thermal antimatter engine (LIPTHANE). LIPTHANE incorporates xenon as its fluid for heat conversion given the high cross-section of xenon with regards to radiation [22]. Xenon is incorporated into non-chemical applications, such as electric propulsion. However, xenon has a considerable atomic mass, especially when compared to hydrogen. Traditional rocket systems can improve their performance, such as specific impulse, through incorporating hydrogen fuel rich mixture ratios. Respective of performance analysis relations, a lower molecular weight is correlated with a higher performance in terms of specific impulse [23] [24].

The selected propulsive fluid for the photofission pulsed space propulsion system is hydrogen. Two scenarios for the hydrogen propulsion strategy are considered for performance analysis, such as only hydrogen and hydrogen with a tungsten plate for high cross section of radiation absorption to supplement the heating of hydrogen. LeMoyne and Mastroianni have successfully demonstrated both of these configurations from a performance perspective for ultra-intense laser derived antimatter propulsion [2].

A representative preliminary configuration of the photofission pulsed space propulsion system is presented by the experimental methods of Schwoerer et al. An ultra-intense laser targets two sheets of tantalum as intermediary before the objective target of uranium-238. The first tantalum sheet is $50 \mu \mathrm{m}$ thick and the second $1 \mathrm{~mm}$ thick. The first sheet generates a hot plasma, and the second sheet emits bremsstrahlung photons that interact with the uranium-238 nuclei producing photofission [14]. Each photofission event progressively advances the thermal load of the hydrogen fluid for a pulsed propulsion configuration.

\section{Fundamental Analysis and Performance Results}

The objective of the fundamental analysis for the photofission pulsed space propulsion system is to enable the perspective of preliminary proof of concept. As photofission becomes a better characterized phenomena, more sophisticated analyses can be applied. As previously defined, each photofission event is approximated to release $200 \mathrm{MeV}$ of energy [5]. $7 \times 10^{4}$ fissions per joule of ultra-intense laser are incorporated to scale the laser requirements [13] [14]. An energy balance is derived to reach a $2000 \mathrm{~K}$ thermal threshold for the hydrogen fluid. The steady state performance parameters of the photofission pulsed space propulsion system are then derived.

Prior to commencing photofission the propulsive fluid is approximated to be $300 \mathrm{~K}$. More advanced analysis techniques can later be applied to determine more representative reference temperatures for spacecraft. Table 1 summarizes the conditions of a closed chamber scenario. Table 2 presents the tungsten and hydrogen material properties.

The energy balance for the number of photofission events is defined by Equation (2). Based on experimentation $7 \times 10^{4}$ fissions per joule of laser energy with uranium-238 as a target is assumed. Based on the intrinsic assumptions each photofission event yields $2.2 \times 10^{-6} \mathrm{~kJ}$ per kJ of ultra-intense laser activity. The chamber is assumed to be adiabatic.

$$
Q_{\text {photofission }}=\left(m_{\text {hydrogen }} c_{p \text { (hydrogen })}+m_{\text {tungsten }} c_{\text {tungsten }}\right)\left(T_{f}-T_{i}\right)
$$

Table 1. Closed chamber conditions.

$\begin{array}{cc}\text { Hydrogen temperature (K) } & 2000 \\ \text { Hydrogen pressure (atm) } & 30 \\ \text { Hydrogen mass (kg) } & 0.01 \\ \text { Tungsten plate mass (kg) } & 0.1\end{array}$

Table 2. Material properties of tungsten and hydrogen at $2000 \mathrm{~K}$.

\begin{tabular}{cc}
\hline Tungsten specific heat $(\mathrm{kJ} / \mathrm{kg}-\mathrm{K})$ & 0.18 \\
Hydrogen constant pressure specific heat $(\mathrm{kJ} / \mathrm{kg}-\mathrm{K})$ & 17.1 \\
Hydrogen specific heat ratio & 1.2 \\
\hline
\end{tabular}

[2] [20] [24] [25]. 
$m_{\text {hydrogen }}:$ Mass of hydrogen

$c_{p \text { (hydrogen) }}$ : Constant pressure specific heat of hydrogen

$m_{\text {tungsten }}:$ Mass of tungsten

$c_{\text {tungsten }}:$ Specific heat of tungsten

$T_{f}$ : Final temperature

$T_{i}$ : Intial temperature

The photofission pulsed space propulsion system utilizes a closed chamber of hydrogen for propulsive fluid, which is heated by photofission. A pulse of thrust is generated after the hydrogen temperature reaches a threshold of $2000 \mathrm{~K}$. Heat addition on the scale of $356 \mathrm{~kJ}$ is required to achieve this threshold. The supporting ultraintense laser systems would require an energy source of $1.6 \times 10^{8} \mathrm{~kJ}$ in order to produce a sufficient quantity of photofission events to heat the hydrogen propulsive fluid to $2000 \mathrm{~K}$.

A $0.1 \mathrm{~kg}$ plate of tungsten that provides enhanced cross-section for absorbing the photofission energy is incorporated in the alternative configuration. The tungsten plate necessitates an additional $31 \mathrm{~kJ}$ of photofission derived heat addition for a total requirement of $387 \mathrm{~kJ}$. With respect to the contingency configuration $1.7 \times 10^{8}$ $\mathrm{kJ}$ of ultra-intense laser energy are required for a hydrogen temperature of $2000 \mathrm{~K}$.

The performance parameters of the photofission pulsed space propulsion system are derived through steady state fundamental propulsion analysis. Both propulsion configurations incorporate 10 grams of hydrogen with a corresponding pressure of $30 \mathrm{~atm}$ and a temperature of $2000 \mathrm{~K}$. Therefore, their propulsion performance attributes are equivalent. A mass flow of 1 gram/second is applied, which is representative of previous work by LeMoyne and Mastroianni [2] [20]. Isentropic analysis is applied for the steady state phase of the expansion through the nozzle to derive specific impulse.

$$
I_{s p}=\frac{1}{g}\left\{\frac{2 \gamma R T_{o}}{\gamma-1}\left[1-\left(\frac{p_{e}}{p_{o}}\right)^{(\gamma-1) / \gamma}\right]\right\}^{1 / 2}
$$

$I_{s p}$ : Specific impulse

$g$ : Gravity

$R$ : Specific gas constant

$T_{o}$ : Stagnation temperature of chamber

$p_{o}$ : Stagnation pressure of chamber

$p_{e}$ : Exit pressure

$\gamma$ :Specific heat ratio [23]

The isentropic analysis assumes the propulsive fluid is expanding into a space environment. Therefore, the exhaust pressure is assumed negligible. The photofission pulsed space propulsion system supported by an ultraintense laser produces a thrust of $10 \mathrm{~N}$ and a specific impulse of 1019 seconds.

The photofission pulsed space propulsion concept enables the decoupling of the energy source and the propulsion system. For example, the energy source could be from a terrestrial position, such as a nuclear reactor linked to an ultra-intense laser, and the propulsion system could be integrated with a distant spacecraft. Given the inherent feature of the laser, the propulsion system could be astronomically remote, such as 3 AU or even 30 $\mathrm{AU}$, from the Earth bound terrestrial power system and ultra-intense laser. Preliminary test and evaluation could be attempted with the experimental procedures of Cowan et al., Ledingham et al., and Schwoerer et al. with photofission heating a hydrogen propulsive fluid source [12]-[14].

\section{Feasibility of Photofission Pulsed Space Propulsion}

The objective of the conceptual architecture is to present the characteristics of a pulsed propulsion system from a fundamental performance analysis perspective. Other ultra-intense laser applications for advanced propulsion configurations, such as the pulsed positron antimatter space propulsion system by LeMoyne and Mastroianni, push the boundaries of the state of the art [2]. With the rampant evolutionary trends, the attributes of ultra-intense laser technology are expected to considerably improve. Advanced regenerative cooling systems may be necessary to ameliorate the extreme thermal loads applied to the target materials during ultra-intense laser pulsing. Regenerative cooling technologies have been successfully applied to other classes of rocket propulsion [24].

Other space propulsion configurations have been conceptually defined, such as the laser propelled light sail, 
which uses a laser beam to enable propulsion [26]. Some of these systems have advocated continuous power budgets on the order of $1 \mathrm{PW}$, which are comparable to the capacity of all civilization [27] [28]. Laser propelled light sails require years of focused targeting. Even in light of the substantial power budgets, they have been published under the scope of future technology applications [29].

\section{Conclusions}

A photofission pulsed space propulsion system architecture is presented and advocated from the perspective of preliminary fundamental performance analysis. Nuclear fission presents considerable opportunity in light of the amount of energy released per fission event. Historically there have been multiple attempts to apply nuclear fission in the context of a propulsion system. However, these attempts have been limited by complexity, such as controlling the inherent neutron flux. Photofission enables a unique capability of a more controlled form of nuclear fission. Ultra-intense lasers can elicit photofission with an intermediate material, such as tantalum, and a pending uranium target. Photofission has been demonstrated from an assortment of heavy nuclei candidates, such as thorium, lead, and naturally occurring isotopes of uranium.

A pulsed space propulsion configuration has been demonstrated with regards to fundamental performance analysis using hydrogen as a propulsive fluid and an alternate configuration integrating a tungsten plate. Both configurations demonstrated the capacity to heat hydrogen to a temperature of $2000 \mathrm{~K}$. Using steady state conditions for isentropic relations a thrust of $10 \mathrm{~N}$ and a specific impulse of 1019 seconds were determined.

A major design observation is the decoupling of the energy source and the propulsive system. The energy source and laser can potentially be terrestrially positioned with the respective photofission pulsed space propulsion system situated in a deep space context. Future configuration advances should address the extreme thermal loading about the ultra-intense laser target with advanced cooling techniques. Laser technologies are highly anticipated to rapidly advance enabling capabilities for the domain of photofission pulsed space propulsion.

\section{References}

[1] LeMoyne, R. (2012) Fundamental Architecture and Analysis of Ramjet Propulsion System Utilizing In-Situ Laser Derived Antimatter. Proceedings of 18th AIAA/3AF International Space Planes and Hypersonic Systems and Technologies Conference, Tours, September 2012.

[2] LeMoyne, R. and Mastroianni, T. (2014) Fundamental Architecture and Analysis of an Antimatter Ultra-Intense Laser Derived Pulsed Space Propulsion System. Journal of Applied Mathematics and Physics, 2, 10-18.

[3] LeMoyne, R. and Mastroianni, T. (2014) Advanced Concept Ramjet Propulsion System Utilizing In-Situ Positron Antimatter Derived from Ultra-Intense Laser with Fundamental Performance Analysis. Journal of Applied Mathematics and Physics, 2, 19-26.

[4] LeMoyne, R. and Mastroianni, T. (2014) Fundamental Architecture and Analysis of an Antimatter Ultra-Intense Laser Derived Combined Cycle Ramjet-Rocket Propulsion System. Proceedings of 19th AIAA International Space Planes and Hypersonic Systems and Technologies Conference, Atlanta, June 2014.

[5] Serway, R.A., Moses, C.J. and Moyer, C.A. (1989) Modern Physics. Saunders College, Philadelphia.

[6] Serway, R.A. (1990) Physics for Scientists and Engineers. Saunders College, Philadelphia.

[7] Robbins, W.H. and Finger, H.B. (1991) An Historical Perspective of the NERVA Nuclear Rocket Engine Technology Program. NASA Contractor Report 187154/AIAA-91-3451, NASA Lewis Research Center, Cleveland.

[8] Howe, S.D. (1985) Assessment of the Advantages and Feasibility of a Nuclear Rocket for a Manned Mars Mission. Proceedings from Manned Mars Mission Workshop, NASA Marshal Space Flight Center, Huntsville, Alabama, June 1985.

[9] Dyson, F.J. (1968) Interstellar Transport. Physics Today, 21, 41-45. http://dx.doi.org/10.1063/1.3034534

[10] US Department of Energy (1993) DOE Fundamentals Handbook Nuclear Physics and Reactor Theory. DOE-HDBK1019/2-93.

[11] Bereznai, G.T. and Harvel, G. (2011) Introduction to CANDU Systems and Operation. Workshop on Nuclear Power Plant Simulators, University of Ontario Institute of Technology, Oshawa.

[12] Ledingham, K.W., Spencer, I., McCanny, T., Singhal, R.P., Santala, M.I., Clark, E., Watts, I., Beg, F.N., Zepf, M., Krushelnick, K., Tatarakis, M., Dangor, A.E., Norreys, P.A., Allott, R., Neely, D., Clark, R.J., Machacek, A.C., Wark, J.S., Cresswell, A.J., Sanderson, D.C. and Magill, J. (2000) Photonuclear Physics When a Multiterawatt Laser Pulse Interacts with Solid Targets. Physical Review Letters, 84, 899-902. http://dx.doi.org/10.1103/PhysRevLett.84.899

[13] Cowan, T.E., Hunt, A.W., Phillips, T.W., Wilks, S.C., Perry, M.D., Brown, C., Fountain, W., Hatchett, S., Johnson, J., 
Key, M.H., Parnell, T., Pennington, D.M., Snavely, R.A. and Takahashi, Y. (2000) Photonuclear Fission from High Energy Electrons from Ultraintense Laser-Solid Interactions. Physical Review Letters, 84, 903-906. http://dx.doi.org/10.1103/PhysRevLett.84.903

[14] Schwoerer, H., Ewald, F., Sauerbrey, R., Galy, J., Magill, J., Rondinella, V., Schenkel, R. and Butz, T. (2003) Fission of Actinides Using a Tabletop Laser. EPL (Europhysics Letters), 61, 47-52. http://dx.doi.org/10.1209/epl/i2003-00243-1

[15] Kavouras, J.G. (2014) Detection and Simulation of Delayed $\gamma$-Rays from Photofission. Master's Thesis, Department of Civil and Environmental Engineering (Nuclear Engineering), University of Utah, Salt Lake City.

[16] Pommé, S., Jacobs, E., Persyn, K., De Frenne, D., Govaert, K. and Yoneama, M.L. (1993) Excitation Energy Dependence of Charge Odd-Even Effects in the Fission of 238U Close to the Fission Barrier. Nuclear Physics A, 560, 689714. http://dx.doi.org/10.1016/0375-9474(93)90041-U

[17] Cetina, C., Berman, B.L., Briscoe, W.J., Cole, P.L., Feldman, G., Heimberg, P., Murphy, L.Y., Philips, S.A., Sanabria, J.C., Crannell, H., Longhi, A., Sober, D.I. and Kezerashvili, G.Y. (2000) Photofission of Heavy Nuclei at Energies up to $4 \mathrm{GeV}$. Physical Review Letters, 84, 5740-5743. http://dx.doi.org/10.1103/PhysRevLett.84.5740

[18] Cetina, C., Heimberg, P., Berman, B.L., Briscoe, W.J., Feldman, G., Murphy, L.Y., Crannell, H., Longhi, A., Sober, D.I., Sanabria, J.C. and Kezerashvili, G.Y. (2002) Photofission of Heavy Nuclei from 0.2 to $3.8 \mathrm{GeV}$. Physical Review C, 65, Article ID: 044622-1-16. http://dx.doi.org/10.1103/PhysRevC.65.044622

[19] Naik, H., Carrel, F., Kim, G.N., Laine, F., Sari, A., Normand, S. and Goswami, A. (2013) Mass Yield Distributions of Fission Products from Photo-Fission of 238U Induced by 11.5 - 17.3 MeV Bremsstrahlung. The European Physical Journal A, 49, 1-23. http://dx.doi.org/10.1140/epja/i2013-13094-7

[20] LeMoyne, R. (2006) Fundamental Analysis of Radioisotope Propulsion. Proceedings of Space 2006, San Jose, September 2006.

[21] Williams, G.J., Jenkins, R.M. and Rose, M.F. (1994) Preliminary Design of a Space Propulsion System Utilizing Stored Thermal Energy. Journal of Propulsion and Power, 10, 32-39. http://dx.doi.org/10.2514/3.23708

[22] Vulpetti, G. (1987) A Further Analysis about the Liquid-Propellant Thermal Antimatter Engine Design Concept. Acta Astronautica, 15, 551-555. http://dx.doi.org/10.1016/0094-5765(87)90155-X

[23] Anderson, J.D. (1989) Introduction to Flight. McGraw-Hill, New York.

[24] Sutton, G.P. (1992) Rocket Propulsion Elements. Wiley-Interscience, New York.

[25] Turns, S.R. (1996) An Introduction to Combustion: Concepts and Applications. McGraw-Hill, New York.

[26] Harris, D., Bille, M. and Reed, L. (2003) Future Space Transportation Technology: Prospects and Priorities. Proceedings of Space 2003, Long Beach, September 2003.

[27] Frisbee, R.H. (2003) Advanced Propulsion for the XXI Century. Proceedings of AIAA/ICAS International Air and Space Symposium and Exposition, Dayton, July 2003.

[28] Frisbee, R.H. and Leifer, S.D. (1998) Evaluation of Propulsion Options for Interstellar Missions. Proceedings of 34th AIAA, ASME, SAE, and ASEE, Joint Propulsion Conference and Exhibit, Cleveland, July 1998.

[29] Andrews, D.G. (2004) Interstellar Propulsion Opportunities Using Near-Term Technologies. Acta Astronautica, 55, 443-451. http://dx.doi.org/10.1016/j.actaastro.2004.05.038 